\title{
Análise do gasto energético em crianças hígidas e com distrofia muscular de Duchenne nos ambientes aquático e terrestre
}

\author{
Analysis of energy expenditure in children with Duchenne muscular \\ dystrophy and healthy children in aquatic and land environments
}

\section{RESUMO}

Introdução: A Distrofia Muscular de Duchenne é uma doença progressiva, que ocasiona o comprometimento funcional.

Metodologia: Para a avaliação do Índice de Gasto Energético dos pacientes com Distrofia Muscular de Duchenne e indivíduos hígidos foi aplicado o Teste de Caminhada de 6 minutos no solo e na água. Participaram do estudo 28 crianças do sexo masculino, sendo 20 do grupo controle idade média 10,45 $\pm 0,49$ e 8 crianças com Distrofia Muscular de Duchenne idade média de 10,75 $\pm 0,43$ anos.

Resultados: No solo, o grupo controle andou uma distância maior comparado ao grupo com Duchenne $(\mathrm{p}=0,001)$. Os grupos apresentaram a média muito próxima da distância percorrida na água, sendo assim, não houve diferença na distância percorrida entre os dois grupos na água $(\mathrm{p}=0,837)$, no IGE podemos observar que o grupo Duchenne obteve valores maiores em ambiente terrestre e aquático $(\mathrm{p}=0,001)$.

Discussão: As crianças com Duchenne necessitam realizar um esforço extra para a execução da marcha. No ambiente aquático, ocorre aumen-

Kaitiana Martins Da SiLva ${ }^{\mathrm{I}}$ Marilia Escudero Cecconi ${ }^{\mathrm{II}}$ Ricardo Cristian Hengles ${ }^{\text {III }}$ MARIANA BETTINIVERDianI ${ }^{\text {IV }}$ Fernanda Moraes Roccov Douglas Martins Braga ${ }^{\text {VI }}$ 'Universidade Federal de São Paulo (UNIFESP), Santos/SP - Brasil "Universidade de São Caetano do Sul (USCS), São Caetano/SP-Brasil. "IIUniversidade do Estado de São Paulo (USP), São Paulo/SP-Brasil.

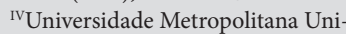
das (FMU), São Paulo/SP-Brasil. vUniversidade de Taubaté (UNITAU), Taubaté /SP- Brasil. ${ }^{\mathrm{V}}$ Universidade Federal do Estado de São Paulo (UNIFESP), São Paulo / SP-Brasil. to do comprimento de passo, e talvez esse fato possa ser explicado pela diminuição da atividade muscular e aumento do gasto energético.

Conclusão: A marcha na água e solo diferem, pois na água a distância percorrida é menor e o índice de gasto energético é maior. Os pacientes com Duchenne no solo percorreram uma distância menor, e na água apresentaram um Índice de Gasto Energético maior.

Palavras-chave: Distrofia Muscular de Duchenne. Marcha. Metabolismo energético. Hidroterapia.

\begin{abstract}
Introduction: Duchenne muscular dystrophy is a progressive disease which leads to a progressive functional commitment.

Methodology: For the evaluation of the Energy Expenditure Index of the patients with Duchenne dystrophy and the healthy ones were used the 6-minute Walk Test in the water and ground environments. Participants were 28 male children, 20 of them from the control group mean age 10.45 and 8 from the Duchenne group mean age of 10.75 years.

Results: On the ground the control group walked a larger distance in comparison with Duchenne group $(p=0,001)$. The groups presented a mean very close to the distance in the water, so there was no difference
\end{abstract}


in the distance the two groups in the water $(\mathrm{p}=0.837)$. In the IGE observe the Duchenne group obtained higher values in terrestrial and aquatic environment $(\mathrm{P}=0.001)$. Discussion: Children with Duchenne need to make extra effort to perform gait. In the aquatic environment there is an increase in the length of step, perhaps this fact can be explained by the decrease of the muscular activity and increase of the energy expenditure.

Conclusion: We can conclude that the walking at the aquatic and terrestrial environments is different, because at the aquatic environment the walked distance was shorter and the Energy Expenditure Index was higher in both groups, the patients with Duchenne at the terrestrial walked a shorter distance and presented an energy expenditure index higher at aquatic environment.

Key-words: Duchenne Muscular Dystrophy. Walking. Energetic Metabolism. Hydrotherapy.

\section{INTRODUÇÃO}

A Distrofia Muscular de Duchenne (DMD) é uma doença progressiva de origem genética, caracterizada pela ausência da proteína distrofina, que leva a uma perda progressiva of functional muscle fibers with mus-das fibras musculares com comprometimento funcional e consequente perda da marcha no início da adolescência (entre 9 e 13 anos de idade) e a seguir, a restrição à cadeira de rodas. Tal fator leva a uma maior perda da força muscular devido à imobilidade, resultando em complicações respiratórias e aumento de peso, que interferem nos aspectos físicos e psicológicos. . $^{1,2,3,4}$

A habilidade de andar é o mais comum de todos os movimentos humanos. Permanecer ereto é uma das características determinantes do homem. $\mathrm{O}$ ato de andar é um evento contínuo que se constitui em transferir o peso de um membro inferior para o outro, com o objetivo de deslocamento.,

Dependendo do ambiente em que a deambulação ocorre, no caso, o meio aquático, outras forças físicas estão presentes, como a de arrasto e empuxo. Elas podem alterar o padrão da marcha, facilitar ou dificultar alguns aspectos no qual irá exigir uma demanda de energia diferente para executar as tarefas. ${ }^{7}$

O gasto energético é analisado pela atividade metabólica de massa magra e pela atividade física. Na DMD, a composição da massa magra é alterada porque há uma destruição progressiva do músculo esquelético, seguido de uma substituição desse tecido por tecido adiposo e tecido fibroso. O gasto energético diário nessas crianças pode ser afetado principalmente pela composição corporal, ocasionando maior demanda de energia para realizar atividades de rotina. ${ }^{8}$

Um programa de reabilitação interdisciplinar é essencial para pacientes com DMD, na tentativa de preservação da sua independência funcional, inserção social, familiar e profissional. A fisioterapia aquática é mais um recurso da reabilitação interdisciplinar com o diferencial de possibilitar e facilitar movimentos que muitas vezes não são mais possíveis no atendimento na fisioterapia em solo, porém, existe uma escassez de estudos no ambiente aquático com a população DMD. Sendo assim, é fundamental o conhecimento das variáveis cinemáticas na realização da marcha nos ambientes aquático e terrestre, para ajudar a entender o funcionamento motor quando em interação com esses meios, e no direcionamento do tratamento. ${ }^{9,10,7}$

Pensando nessas considerações, estabelecemos como objetivo deste trabalho comparar o gasto energético de crianças hígidas e crianças com DMD nos ambientes aquático e terrestre. 


\section{MATERIAL E MÉtodos}

Este é um estudo transversal que foi realizado no setor de fisioterapia aquática da Associação de Assistência à Criança Deficiente (AACD) de São Paulo, no Brasil. O trabalho foi aprovado pelo Comitê de Ética em Pesquisa da mesma instituição, com parecer $\mathrm{n}^{\circ}$. 63.940. A execução deste estudo está baseada na resolução no ${ }^{\circ}$. 466/12 do Conselho Nacional de Saúde.

Participaram do estudo pacientes com diagnóstico médico de DMD e crianças hígidas.

Os critérios de inclusão do grupo com DMD (GDMD) foram pacientes deambuladores, diagnóstico de DMD registrado em prontuário clínico, colaborativo para a realização dos testes, que não tivessem doenças associadas, e que não fizessem parte de programas de reabilitação. Os critérios de exclusão foram pacientes não deambuladores, complicação respiratória há menos de um mês da avaliação, cardiopatia não controlada, não colaborativa para a realização dos testes, que estivessem enquadrados em programas de reabilitação e com presença de afecções dermatológicas que contraindicassem a entrada em piscina.

Quanto aos critérios de inclusão do grupo controle (GC): crianças hígidas, com média de idade próxima ao do GDMD, colaborativo para a realização dos testes e sem histórico de complicações respiratórias. Quanto aos critérios de exclusão: crianças com alguma doença de base, média de idade diferente do grupo GDMD, não colaborativo para a realização dos testes, com histórico de doenças respiratórias, alteração motora e com presença de afecções dermatológicas que contraindicassem a entrada em piscina.

Foram selecionadas 20 crianças para o GC e oito crianças para o GDMD, sendo todas do sexo masculino. GC com idade média de 10,45 $\pm 0,49$ anos e GDMD com média de idade de 10,75 $\pm 0,43$ anos (Tabela 1 ).

Tabela 1 - Características Sociodemográficas e Clínicas

\begin{tabular}{|c|c|c|c|c|}
\hline & \multicolumn{2}{|c|}{ Grupo Controle } & \multicolumn{2}{|c|}{$\begin{array}{c}\text { Grupo distrofia } \\
\text { Muscular de } \\
\text { Duchenne }\end{array}$} \\
\hline Ensino fundamental & & & & \\
\hline $5^{\circ}$ ano & 20 & $100 \%$ & 04 & $50 \%$ \\
\hline $4^{\circ}$ ano & - & - & 03 & $37,5 \%$ \\
\hline $3^{\circ}$ ano & - & - & 01 & $12,5 \%$ \\
\hline Idade & & & & \\
\hline 10 anos & 9 & $45 \%$ & 2 & $25 \%$ \\
\hline 11 anos & 11 & $55 \%$ & 6 & $75 \%$ \\
\hline Faz uso de medicações & - & - & 5 & $62,5 \%$ \\
\hline $\begin{array}{l}\text { Faz uso de ressuscitador } \\
\text { manual }\end{array}$ & - & - & 4 & $50 \%$ \\
\hline
\end{tabular}


Os pais das crianças estavam cientes dos objetivos e procedimentos deste estudo e assinaram o Termo de Consentimento Livre e Esclarecido. As avaliações foram realizadas em ambiente aquático e terrestre pelo mesmo examinador, obedecendo aos mesmos parâmetros para os dois ambientes.

O instrumento de avaliação utilizado foi o teste de caminhada de 6 minutos (TC6). No ambiente terrestre, foi realizado num corredor plano, com superfície lisa e com comprimento de 30 metros em que o início e o final do trajeto foram delimitados com cones de sinalização. No ambiente aquático, foi realizado em uma piscina com 6 metros de comprimento e sem inclinação. Foram verificadas a frequência cardíaca (FC) e a frequência respiratória (FR) no início e no final do teste. ${ }^{11}$ Como medida de segurança utilizamos a oximetria de pulso para mensurar a saturação de oxihemoglobina (aparelho tipo Clip Ônix 9500 Nonim $^{\circledast}$ ), com o valor de $90 \%$ como referência, em que o teste seria imediatamente interrompido se estivesse abaixo desse valor. ${ }^{12,13}$ No ambiente aquático, o processo xifoide foi considerado o ponto de referência de imersão e a temperatura da água manteve-se $32^{\circ} \mathrm{C}$. Foi feito um intervalo de 30 minutos entre os testes com o objetivo de retornar aos parâmetros basais e eliminar o fator fadiga. Após a aplicação do teste para calcular o índice de gasto energético (IGE), utilizamos a fórmula que é constituída por: FC final - FC inicial dividida pela velocidade (metros/segundos), sendo expresso em batimentos/ minuto (bpm). ${ }^{14}$

\section{ANÁlise estatística:}

Os dados da distância percorrida (metros) e IGE no ambiente aquático e terrestre durante o TC6 foram avaliados por análise não paramétrica de Wilcoxon, para comparar os dados em ambiente aquático e terrestre intragrupos. As comparações entre o GC e GDMD foram realizadas por análise não paramétrica e não pareada de Mann-Whitney considerando um intervalo de confiança de 95\%. Foram utilizados os softwares: SPSS V17, Minitab 16 e Excel Office 2010, usando o nível de significância de $\mathrm{p}<0,05$.

\section{Resultados}

Com relação à distância percorrida nos ambientes (terrestre e aquático), notamos que no ambiente terrestre o GC andou uma distância maior comparado ao GDMD ( $p$ $=0,001$ ) (Figura 1). No ambiente aquático, os grupos apresentaram uma média muito próxima na distância percorrida, sendo assim, não houve diferença significativa na distância percorrida entre os dois grupos no ambiente aquático $(\mathrm{p}=0,837)$.

Figura 1: comparação entre os grupos quanto a distância percorrida em ambiente terrestre e aquático (Mann-Whitney $\mathrm{p}<0,05$ ).

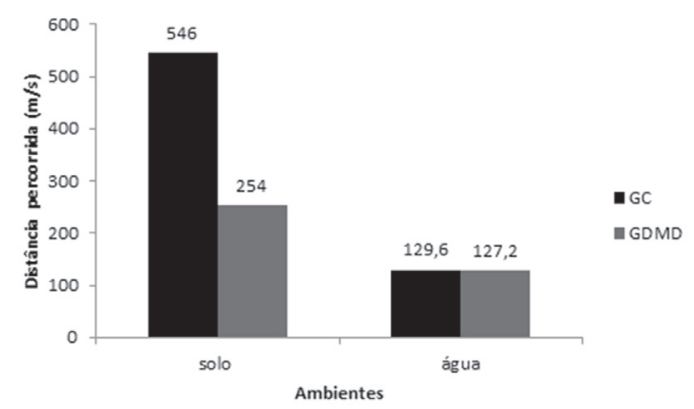


Figura 2: comparação entre os grupos quanto ao índice de gasto energético em ambiente terrestre e aquático (Mann-Whitney p < 0,05).

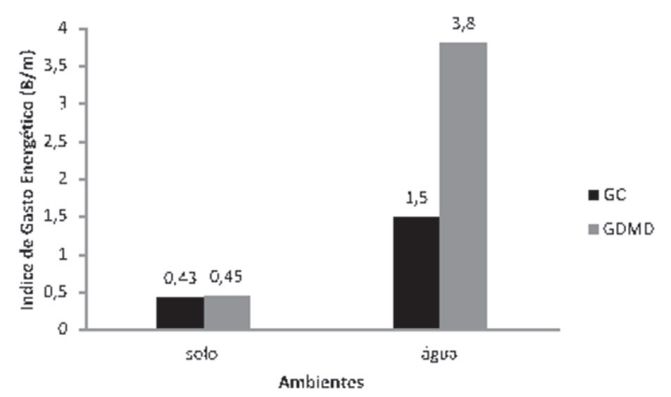

$\mathrm{Na}$ análise de comparação dos grupos GC e GDMD quanto ao IGE podemos observar que o grupo GDMD obteve valores maiores tanto em ambiente terrestre quanto em ambiente aquático. Porém, apresentou uma diferença significativa apenas quando comparado o grupo GDMD com o grupo GC em ambiente aquático ( $\mathrm{p}=0,001)$.

Ao analisar ambos os grupos, notamos que existe diferença significativa durante a marcha no ambiente aquático e terrestre ( $p$ $=0,001)$ para as variáveis distância e IGE. Observamos que a distância no ambiente aquático é sempre menor do que no ambiente terrestre. Já com o IGE, ocorre o inverso, ou seja, os valores em ambiente aquático são sempre maiores do que no ambiente terrestre $(\mathrm{p}=0,001)$ (Tabela 2).

Tabela 2: Compara ambiente terrestre e aquático por grupo e ambos os grupos para distância e IGE.

\begin{tabular}{|c|c|c|c|c|c|c|c|c|c|}
\hline \multirow[t]{2}{*}{ Variáveis } & \multicolumn{3}{|c|}{ GC } & \multicolumn{3}{|c|}{ GDMD } & \multicolumn{3}{|c|}{ Ambos os grupos } \\
\hline & Solo & Água & & Solo & Água & & Solo & Água & \\
\hline $\begin{array}{l}\text { Distância } \\
(\mathrm{m} / \mathrm{s})\end{array}$ & $\begin{array}{c}546,0 \\
(520,0 / 578,5)\end{array}$ & $\begin{array}{c}129,6 \\
(124,1 / 131,3)\end{array}$ & ${ }^{*} 0,001$ & $\begin{array}{c}254,0 \\
(212,0 / 263,8)\end{array}$ & $\begin{array}{c}127,2 \\
(115,2 / 139,4)\end{array}$ & ${ }^{\star} 0,012$ & $\begin{array}{c}520,0 \\
(278,0 / 572,0)\end{array}$ & $\begin{array}{c}129,6 \\
(118,8 / 132,5)\end{array}$ & ${ }^{\star} 0,001$ \\
\hline (bpm) & $\begin{array}{c}0,43 \\
(0,24 / 0,53)\end{array}$ & $\begin{array}{c}1,50 \\
(1,09 / 1,90)\end{array}$ & ${ }^{*} 0,001$ & $\begin{array}{c}0,45 \\
(0,33 / 0,49)\end{array}$ & $\begin{array}{c}3,80 \\
(3,38 / 4,04)\end{array}$ & ${ }^{*} 0,012$ & $\begin{array}{c}0,45 \\
(0,24 / 0,52)\end{array}$ & $\begin{array}{c}1,89 \\
(1,35 / 3,23)\end{array}$ & ${ }^{*} 0,001$ \\
\hline
\end{tabular}

Legenda: Mediana ( $1^{\circ}$ Quartil/3 $/ 3^{\circ}$ uartil) e p-valor da comparação dos grupos GDMD e GC, e ambos os grupos com significância estatística, em solo e em água* vs. (Wilcoxon, $\mathrm{p}<0,05$ ).

\section{DISCUSSÃo}

Esta pesquisa apresenta como principal objetivo verificar o IGE no ambiente aquático e terrestre em crianças com DMD e hígidas, procuramos montar um grupo mais homogêneo possível, pois a idade e o está- gio da doença são fatores relevantes e podem influenciar na pesquisa, uma vez que estamos estudando uma doença progressiva com diferentes estágios. ${ }^{15}$

Optamos pela escolha do TC6 que, segundo a literatura, ${ }^{23}$ é o teste mais apropriado para refletir a capacidade física durante 
a análise da marcha das crianças com DMD e Distrofia Muscular de Becker. Os pesquisadores notaram que na avaliação em solo com TC6, elas apresentam uma velocidade de marcha e distância percorrida menor em comparação com crianças da mesma faixa etária e hígidas. Essa diferença está relacionada com menor comprimento do passo. ${ }^{16,17}$

Sabe-se que crianças com DMD com 4 anos de idade apresentaram um déficit de $20 \%$ no TC6 em relação às crianças hígidas , e esse déficit permanece estável até meados do final da infância, entre as idades de $10 \mathrm{e}$ 12 anos. A partir desse período, podem diminuir o desempenho da marcha em relação aos saudáveis em $20 \%$ ao ano ou mais. ${ }^{17}$ Esses achados coincidem com o nosso estudo que, apesar de não termos realizado a contagem dos passos, o GC percorreu uma distância maior que o GDMD.

As crianças com DMD necessitam realizar um esforço extra para a execução da marcha, devido ao maior recrutamento de unidades motoras e a utilização de músculos que se adaptam para compensar a fraqueza muscular usada para o deslocamento, isto ocorre, pois é necessário ajustes constantes durante a deambulação, ${ }^{18}$ resultando em uma menor velocidade de marcha. ${ }^{19}$ Esse dado se reflete em nosso estudo em que o GDMD andou uma distância menor em solo comparado ao GC.

$\mathrm{O}$ andar no ambiente terrestre promove uma velocidade de marcha diferente quando comparado com o ambiente aquático, mesmo na população sem afecções neurológicas, sendo atribuídas a diversos fatores: força de arrasto durante o movimento, diminuição do peso corporal gerado pelo empuxo e consequente diminuição da velocidade da marcha. ${ }^{20}$ No ambiente aquático ocorre aumento do comprimento de passo, ${ }^{21}$ esses fatos embasam nossos achados, pois, ambos os grupos apresentaram uma diminuição da distância percorrida na água quando comparado ao solo.

As crianças com DMD demandam um gasto energético maior durante as atividades, porém, há uma escassez de estudos voltados para essa população, principalmente em relação à marcha no ambiente aquático. $\mathrm{Na}$ análise do IGE de crianças com DMD, observou-se um menor índice quando comparado com crianças hígidas. Esse fato aconteceu, pois, as crianças com DMD têm diminuição da ativação muscular, porém, essa análise foi feita apenas no ambiente terrestre e em repouso. ${ }^{8}$ Neste estudo, analisamos a atividade da marcha e ocorreu o inverso, as crianças com DMD aumentaram o IGE, pois existe uma maior demanda de ativação muscular no ambiente aquático. Acreditamos que isso aconteceu, pois é necessário produzir uma força de propulsão para superar a força do arrasto ao andar na água, fazendo que as atividades musculares, cardiorrespiratórias e respostas perceptivas sejam superiores aos resultados da marcha em ambiente terrestre, ocorrendo um aumento do IGE nesse ambiente. ${ }^{22}$

$\mathrm{O}$ aumento do gasto energético em crianças com DMD durante a marcha no ambiente terrestre está diretamente relacionado a uma tentativa em manter uma cadência elevada para compensar o menor comprimento de passo e a velocidade de marcha reduzida. ${ }^{21}$

Considerando o nível de imersão em processo xifoide, devemos considerar que neste ocorrerá alívio parcial do peso corporal devi- 
do ao empuxo, mas aumenta a área de atrito durante o deslocamento, necessitando maior demanda de IGE para vencer a força de arrasto, dado observado em ambos os grupos. ${ }^{23}$

A literatura da DMD sugere que os tratamentos mudaram o curso da doença. $\mathrm{O}$ tempo de vida dos pacientes com DMD é mais longo, devido ao empenho de equipes multidisciplinares para melhorar sua qualidade de vida. Alguns estudos sugerem que o uso da fisioterapia para retardar a perda da função motora e atrasar as complicações cardiorrespiratórias tem aumentado o tempo de sobrevivência em pelo menos dez anos. ${ }^{24} \mathrm{~A}$ hidroterapia é mais um recurso dentro do programa de reabilitação, pois os exercícios são de baixo impacto e a água oferece suporte e apoio durante a marcha, ${ }^{25}$ mas sabemos que é importante compreender as alterações funcionais observadas em pacientes com DMD durante o seu desenvolvimento, pois nos direciona no momento de formular a intervenção te- rapêutica para essa população que apresenta característica clínica variável dependendo do estágio da doença. ${ }^{26,27}$

Diversos aspectos devem ser considerados durante a marcha subaquática para a recuperação funcional, como nível de imersão, que é fator determinante acerca das forças que agem durante a marcha, e a posição dos membros superiores que podem causar alterações nos valores das forças atuantes na água. ${ }^{28}$ Esses fatores devem ser considerados em estudos futuros com essa população, por isso sugerimos aumentar a amostra e incluir indivíduos em outros estágios da doença.

\section{Conclusão}

O IGE é significativamente maior nas crianças com DMD quando comparado com as crianças hígidas apenas no ambiente aquático, apresentando uma média próxima no ambiente terrestre.

\section{REFERÊNCIAS}

1. Macdonald C. M. Physical activity, health impairments, and disability in neuromuscular disease. American Journal Physiotherapy Medicine and Rehabilitation, 2002; 81(11): 108-20.

2. Tidball J., Wehling-Henricks M. Evolving therapeutic strategies for Duchenne muscular dystrophy: targeting downstream events. Pediatr Res. 2004; 56(6): 831-41.

3. Hermans MC, Pinto YM, Merkies ISJ, Die-Smulders CEM, Crijns HJGM, Faber CG. Hereditary muscular dystrophies and the heart. Neuromuscul Disord. 2010; 20(8): 479-92.

4. Jansen M., Groot IJM, Geurts ACH, Alfen, N. V. Physical training in boys with Duchenne Muscular Dystrophy: the protocol of the No Use is Disuse study. BMC Pediatrics. 2010; 10(55): 2-15.

5. Ribas DIR, Israel VL, Manfra EF, Araújo CC. Estudo comparativo dos parâmetros angulares da marcha humana em ambiente aquático e terrestre em indivíduos hígidos adultos jovens. Rev Bras Med Esporte. 2007; 13(6): 371-5.

6. Souza PV, Fonta HB, Haupenthal A, Schutz GR, Roesler H. Força de reação do solo durante a marcha de crianças em ambiente aquático. Pediatr Mod. 2010; 46(4): 146-150.

7. Brito RN, Roesler H., Haupenthal A., Souza P. Análise comparativa da marcha humana em solo à subaquática em dois níveis de imersão: joelho e quadril. Braz. J. Phys. Ther. 2004; 8(1):7-12.

8. Zanardi MC, Tagliabue A., Orcesi S., Berardinelli A., Uggetti C., Pichiecchio A. Body composition and energy expenditure in Duchenne muscular dystrophy. European Journal of Clinical Nutrition. 2003; 57: 273-278. 
9. Ribas DIR, Israel VL, Manfra EF, Araújo CC. Estudo comparativo dos parâmetros angulares da marcha humana em ambiente aquático e terrestre em indivíduos hígidos adultos jovens. Rev Bras Med Esporte. 2007; 13(6): 371-5.

10. Souza PV, Fonta HB, Haupenthal A., Chutz GR, Roesler H. Força de reação do solo durante a marcha de crianças em ambiente aquático. Pediatr Mod. 2010; 46(4): 146-150.

11. Temas em revisão. Sociedade Brasileira de Pneumologia e Tisiologia. Disponível em: < http:// www. sbpt.org.br >. Acesso em: 06/07/2016.

12. Chin T., Sawamura S., Fujita H., Nakajima S., Ojima I., Oyabu H., Nagakura Y., Otsuka, H., Nakagawa, A. The efficacy of physiological cost index (PCI) measurement of a subject walking with an Intelligent Prosthesis. Prosthetics and Orthotics International. 1999; 23(1): 45-9.

13. Salyer, J. W. Neonatal and Pediatric Pulse Oximetry. Respiratory Care. 2003; 48(4): 386-396.

14. Salmela LFT, Silva PC, Lima RCM, Augusto ACC, Souza AC, Goulart F. Musculação e condicionamento aeróbio na performance funcional de hemiplégicos crônicos. Actafisiátrica. 2003; 10(2): 54-60.

15. Goemans N., Hauwe M., Wilson R., Klingels K., Buyse G. Ambulatory capacity and disease progression as measured by the 6-minute-walk-distance in Duchenne muscular dystrophy subjects on daily corticosteroids. Neuromuscul Disord. 2013; 23(8): 618-623.

16. Mac Donald C. M. Physical activity, health impairments, and disability in neuromuscular disease. American Journal Physiotherapy. Medicine and Rehabilitation. 2002; 81(11): 108-20.

17. MC Donald C. M., Henricson E. K., Han JJ, Abresch RT, Nicorici A., Elfring GL, Atkinson, L. et al. The 6-minute walk test as a new outcome measure in Duchenne muscular dystrophy Muscle Nerve. 2010; 41: 500-510.

18. Hind D., Parkin J., Whitworth V., et al. Aquatic therapy for boys with Duchenne muscular dystrophy (DMD): an external pilot randomised controlled trial. Pilot Feasibility Stud. 2017; 3(1): 16. doi:10.1186/s40814-017-0132-0.

19. Barela AMF, Stolf SF, Duarte M. Biomechanical characteristics of adults walking in shallow water and on land. Journal of Electromyography and Kinesiology. 2006; 16: 250-256.

20. Carneiro LC, Haupenthal A., Schütz G R, Souza PV, Tavares GMS, Roesler H. Características cinemáticas e dinamométricas da marcha de crianças em ambiente aquático Fisioter. Mov. Curitiba. 2009; 22(3):427-438.

21. Gaudreault N., Gravel D., Nadeau S., Houde S., Gagnon D. Gait patterns comparison of children with Duchenne muscular dystrophy to those of control subjects considering the effect of gait velocity. Gait \& Posture. 2010; 32: 342-347.

22. Masumoto K., Mercer JA. Biomechanics of Human Locomotion in Water: An Electromyographic Analysis. Exerc Sport Sci Rev. 2008; 36(3): 160-9.

23. Haupenthal A., Schütz G R, Hubert M., Dabonneville M., Roesler H. Force analysis of the underwater stationary running. XXV ISBS Symposium. OuroPreto - Brazil. 2007: 103-106.

24. Sá C dos SC de, Fagundes IK, Araújo TB, Oliveira ASB, Fávero FM. The relevance of trunk evaluation in Duchenne muscular dystrophy: the segmental assessment of trunk control. Arq Neuropsiquiatr. 2016; 74(10):791-795. doi: 10.1590/0004-282X20160124.

25. Anziska Y., Inan S. Exercise in neuromuscular disease. Semin Neurol. 2014; 34(5): 542-556. doi: 10.1055/s-0034-1396008.

26. Henricson E., Abresch R., Han JJ, Nicorici A., Goude KE, Elfring G., Reha A., Barth J. MCdonald, C. M. Percent-Predicted 6-Minute Walk Distance in Duchenne Muscular Dystrophy to Account for Maturational Influences. PLOS Currents Muscular Dystrophy. 2012 Edition 1. doi: 10.1371/currents.RRN1297. 
27. Ferreira AVS, Goya PSA, Ferrari R, et al. Comparison of motor function in patients with Duchenne muscular dystrophy in physical therapy in and out of water: 2-year follow-up. Acta Fisiátrica. 2015; 22(2): 51-54. doi:10.5935/0104-7795.20150011

28. Roesler H., Haupenthal A., Schütz GR, SOUZA P. V. Análise das forças de reação do solo na marcha de adultos a 1,3m de imersão. Fisioterapia em Movimento. 2005; 18(4): 21-31.

\section{DAdos dos Autores}

\section{Kaitiana Martins da Silva}

Mestra em Ciência da Saúde pela Universidade Federal do Estado de São Paulo. Santos/SP-Brasil.kaitianafisio@hotmail.com

\section{Marilia Escudero Cecconi}

Graduada em Fisioterapia pela Universidade Metodista de São Paulo. Fisioterapeuta do Setor de Fisioterapia Adulto da Associação de Assistência à Criança Deficiente - Ibirapuera. São Paulo/SP-Brasil. marilia.cecconi@hotmail.com

\section{Ricardo Cristian Hengles}

Mestrando em Ciências da Reabilitação na Universidade de São Paulo. Fisioterapeuta do Setor de fisioterapia infantil da Associação de Assistência à Criança Deficiente - Ibirapuera. São Paulo/SP-Brasil.ricardo_hengles@hotmail.com

\section{Mariana BetTiniverdiani}

Graduada em Fisioterapia pela Universidade Metodista de São Paulo. Fisioterapeuta do Setor de Fisioterapia Aquática da Associação de Assistência à Criança Deficiente - Ibirapuera. São Paulo/SP-Brasil. Mariana_1305hotmail.com

\section{Fernanda Moraes Rocco}

Graduada em Medicina pela Universidade de Taubaté. Médica Fisiatra responsável pela Clínica de Doenças Neuromusculares da AACD - Ibirapuera. São Paulo/SP-Brasil. ferocco@ msn.com

\section{Douglas Martins Braga}

Mestrando em Neurologia e Neurociências na Universidade Federal de São Paulo. Fisioterapeuta do Setor de Fisioterapia Aquática da Associação de Assistência à Criança Deficiente - Ibirapuera. São Paulo/SP-Brasil.douglasbraga78@hotmail.com

Submetido em: 20-02-2017

Aceito em: 28-04-2017 